Copyright by the American Physical Society. Badro, J.; Teter, D. M.|; Downs, R. T.; Gillet, P.; Hemley, R. J.|; Barrat, J. L.,

"Theoretical study of a five-coordinated silica polymorph," Phys. Rev. B 56, 5797 DOI: http://dx.doi.org/10.1103/

PhysRevB.56.5797

PHYSICAL REVIEW B

VOLUME 56, NUMBER 10

1 SEPTEMBER 1997-II

\title{
Theoretical study of a five-coordinated silica polymorph
}

\author{
James Badro \\ Laboratoire de Sciences de la Terre (UMR 5570), Ecole Normale Supérieure de Lyon, \\ 46 allée d'Italie F-69364 Lyon Cedex 07, France \\ David M. Teter \\ Geophysical Laboratory and Center for High Pressure Research, Carnegie Institution of Washington, \\ 5251 Broad Branch Road, N.W., Washington, DC 20015 \\ and Department of Materials Science and Engineering, Virginia Tech, Blacksburg, Virginia 24061
}

Robert T. Downs*

Geophysical Laboratory and Center for High Pressure Research, Carnegie Institution of Washington, 5251 Broad Branch Road, N.W., Washington, DC 20015

Philippe Gillet

Institut Universitaire de France, Laboratoire de Sciences de la Terre (UMR 5570), Ecole Normale Supérieure de Lyon, 46 allée d'Italie F-69364 Lyon Cedex 07, France

Russell J. Hemley

Geophysical Laboratory and Center for High Pressure Research, Carnegie Institution of Washington, 5251 Broad Branch Road, N.W., Washington, DC 20015

Jean-Louis Barrat

Departement de Physique des Materiaux (UMR 5561)-CNRS, Université Claude Bernard, 43 bd. du 11 novembre 1918, F-69622 Villeurbanne Cedex, France

(Received 12 February 1997)

\begin{abstract}
Theoretical calculations are performed to study transformations in silica as a function of nonhydrostatic stress. Molecular-dynamics calculations reveal a crystalline-to-crystalline transition from $\alpha$-quartz to a phase with five-coordinated silicon $\left({ }^{\mathrm{V}} \mathrm{Si}\right)$ at high pressure in the presence of deviatoric stress. The phase, which appears for specific orientations of the stress tensor relative to the crystallographic axes of quartz, is a crystalline polymorph of silica with five-coordinated silicon. The structure possesses $P 3_{2} 21$ space-group symmetry. First-principles calculations within the local-density approximation, as well as molecular dynamics and energy minimization with interatomic potentials, find this phase to be mechanically and energetically stable with respect to quartz at high pressure. The calculated $\mathrm{x}$-ray diffraction pattern and vibrational properties of the phase are reported. Upon decompression, the ${ }^{{ }} \mathrm{Si}$ phase reverts to $\alpha$-quartz through an intermediate fourcoordinated phase and an unusual isosymmetrical phase transformation. The results suggest the importance of application of nonhydrostatic stress conditions in the design and synthesis of novel materials. [S0163-1829(97)04034-4]
\end{abstract}

\section{INTRODUCTION}

The bonding in silica gives rise to a rich variety of structures and physical properties of phases in this system as functions of pressure and temperature. Most of the approximately 30 silica polymorphs are stable at low pressures (e.g., quartz, cristobalite, and tridymite) and can be described by various spatial arrangements of $\mathrm{SiO}_{4}$ tetrahedra. ${ }^{1}$ This fourfold coordination of silicon $\left({ }^{\mathrm{IV}} \mathrm{Si}\right)$ results from strong $s p^{3}$ bonding and the large number of packing sequences is allowed by the soft, deformable, Si-O-Si linkage joining the rather rigid $\mathrm{SiO}_{4}$ units. ${ }^{\mathrm{IV}} \mathrm{Si}$ is the fundamental building block of such technologically important materials as amorphous silica optical waveguides, quartz crystal oscillators, and siliceous molecular sieves. It is also one of the most common structural motifs found in minerals that make up the Earth's crust. At higher pressures, silicon increases its coordination number as in the high-density phase, stishovite. In this case, the $\mathrm{Si}$ atoms are coordinated to six $\mathrm{O}$ atoms ( ${ }^{\mathrm{VI}} \mathrm{Si}$ ) to form a network of $\mathrm{SiO}_{6}$ octahedra, a configuration that gives rise to a more ionic $\mathrm{Si}-\mathrm{O}$ bond. This denser arrangement is found in many of the minerals that make up the Earth's mantle. Four- and six-coordinated silica polyhedra have long been considered fundamental building blocks of minerals, glasses, and melts relevant to the mineralogy and geochemistry of the Earth's interior.

The transformations among the $\mathrm{SiO}_{2}$ polymorphs have been studied thoroughly over the years. ${ }^{2,3}$ The low-pressure 
transformations are not accompanied by changes in Si coordination but instead involve modifications in the manner in which $\mathrm{SiO}_{4}$ tetrahedra are linked. The ${ }^{\mathrm{IV}} \mathrm{Si}$ to ${ }^{\mathrm{VI}} \mathrm{Si}$ coordination changes are only observed experimentally at pressures in excess of $8 \mathrm{GPa}(>700 \mathrm{~K})$ by the formation of stishovite. In the recent years, other transformations have been discovered during room-temperature static compression. ${ }^{3} \alpha$-quartz, the stable form at ambient conditions, undergoes a crystalline-to-crystalline transition at $21 \mathrm{GPa}$ prior to pressure-induced amorphization. ${ }^{4-7}$ Similar transformations have been observed for cristobalite and for the isomorphic forms of quartz, $\mathrm{GeO}_{2}$ and $\mathrm{AlPO}_{4} \cdot{ }^{8-11}$ These transitions are associated with the formation of tetrahedral networks, and at higher pressure, increases in cation coordination number. Recently, it has been shown that stishovite transforms at 50 GPa to a phase having the $\mathrm{CaCl}_{2}$ structure type, which also contains ${ }^{\mathrm{VI}} \mathrm{Si} .{ }^{12}$ Increases in $\mathrm{Si}$ coordination have also been inferred from spectroscopic and diffraction measurements of statically compressed silica glass or quartz amorphized at room temperature. ${ }^{4,5,13-16}$

Although all of the observed stable and metastable crystalline phases of silica consist of either ${ }^{\mathrm{IV}} \mathrm{Si}$ or ${ }^{\mathrm{VI}} \mathrm{Si}$, there has been great interest in the possibility of forming phases in which silicon has fivefold coordination by oxygen $\left({ }^{\mathrm{V}} \mathrm{Si}\right)$. This species has been predicted in theoretical studies of the effects of pressure and temperature on silicate melts and glasses ${ }^{17-27}$ and inferred experimentally by ${ }^{29} \mathrm{Si}$ NMR and vibrational spectroscopy in alkali silicate liquids and glasses. ${ }^{16,28-36}$ Silicon has also been found in fivefold coordination with oxygen in a number of organosilicon compounds. ${ }^{37,38} \mathrm{~V}_{\mathrm{Si}}$ has been proposed to play a role in the dissolution of silicates, ${ }^{39-41}$ the increased diffusivity of liquid $\mathrm{SiO}_{2}$ under pressure, ${ }^{18}$ and viscous flow processes of other silicate melts. ${ }^{30-32}$ It has also been speculated to be an important species in the synthesis of siliceous molecular sieves. ${ }^{37,38}$ Thus, although ${ }^{\mathrm{V}} \mathrm{Si}$ is suspected in high-pressure silicates and may form part of the network of high-pressure amorphous phases, and hypothesized in high-temperature $\mathrm{SiO}_{2}$-rich melts or glasses, no crystalline phases of silica containing ${ }^{\mathrm{V}} \mathrm{Si}$ have yet been observed. The synthesis of a crystalline phase of $\mathrm{SiO}_{2}$ entirely composed of $\mathrm{SiO}_{5}$ units would thus be of interest from the point of view of solid-state physics and chemistry, geophysics, and materials science.

The ability of theoretical calculations to predict the physical properties and energetics of the phases of silica has progressed significantly in recent years (e.g., Refs. 42-45). First-principles electronic structure methods are now capable of accurate predictions of phase transformations and vibrational, and elastic properties, as in the recent prediction of the phase transition in stishovite. ${ }^{12}$ Interatomic potentials are capable of reproducing structures, vibrational properties, elastic properties, equations of state, and phase transitions among the known polymorphs. Simulations with these potentials have predicted that the formation of ${ }^{\mathrm{v}} \mathrm{Si}$ is promoted by high pressures; for $\mathrm{SiO}_{2}$ glass these studies show that ${ }^{\mathrm{IV}} \mathrm{Si}$ species are gradually replaced by ${ }^{\mathrm{V}} \mathrm{Si}$ and ${ }^{\mathrm{VI}} \mathrm{Si}$ with increasing pressure. ${ }^{18,19,27,40}$ Such simulations permit the exploration over a broad range of the effects of pressure, temperature, and deviatoric (differential) stresses on phase stability and physical properties. In fact, recent molecular-dynamics simulations on $\alpha$-quartz under nonhydrostatic stress found evidence for a crystalline phase of $\mathrm{SiO}_{2}$ composed entirely of $\mathrm{SiO}_{5}$ groups. ${ }^{27}$ This structure, designated here as penta- $\mathrm{SiO}_{2}$, was discovered during a series of molecular-dynamics simulations undertaken to determine the effect of nonhydrostatic stresses on the pressure-induced amorphization of $\alpha$-quartz. ${ }^{27}$ In these simulations, the isotropic pressure variable was replaced by the three components of the diagonal stress tensor, and each of these variables was monitored and controlled independently. The process allowed the system to access configurations not otherwise found on isotropic compression of quartz.

In this paper, we present a detailed study of this transformation and this predicted phase of $\mathrm{SiO}_{2}$. As such, the paper includes discussion of the original simulations reported as a short letter in Ref. 27 together with the results of new calculations. The crystal structure is examined in detail using molecular-dynamics (MD) and energy minimization techniques with interatomic potentials, as well as first-principles total-energy methods. These techniques are also used to determine its energetics and physical properties, including simulated atomic positions, $\mathrm{x}$-ray diffraction pattern, and vibrational density of states. We find a continuous transformation upon decompression of penta- $\mathrm{SiO}_{2}$ to $\alpha$-quartz via an intermediate crystalline phase. This is an unusual transformation, as all three crystal structures possess the same $P 3_{2} 21$ space-group symmetry. The study shows that nonhydrostatic pressures applied along carefully chosen crystallographic directions could open up a possibility for synthesizing crystalline phases that cannot otherwise be obtained by application of hydrostatic pressure.

\section{THEORETICAL METHODS}

\section{A. Molecular dynamics}

The molecular-dynamics simulations were carried out in a modified $N, \sigma, T$ isobaric-isothermal canonical ensemble, in which the stresses $\sigma_{x x}, \sigma_{y y}$, and $\sigma_{z z}$ were monitored independently. The van Beest, Kramer, and van Santen (BKS) interatomic potential was used. ${ }^{46}$ The orthorhombic simulation box measured $22.326 \times 30.889 \times 17.945 \AA$ at ambient conditions, with the $z$ direction parallel to the $c$ axis of $\alpha$-quartz, and contained 1440 atoms $\left(480 \mathrm{SiO}_{2}\right.$ units). The system was relaxed within this orthorhombic box and the external stress monitored using Berendsen's scaling independently along the three directions. ${ }^{47}$ The temperature was fixed at $300 \mathrm{~K}$ using Hoover's thermostat and the equations of motion were integrated with a time step of $1.34 \mathrm{fs}$ and the long-range Coulomb interactions were calculated using the Ewald sum method. The maximum radius of the wave vectors sphere in reciprocal space was set to $0.273 \AA^{-1}$, and the Ewald parameter for the real-space part of the long-range interaction was also set to the same value. Each compression was carried out over 50000 steps accounting for a total compression time of 134 ps. Molecular-mechanics simulations under $P 1$ symmetry were also performed using the BKS potential. The results of these calculations were very similar to the MD results obtained with the BKS potential.

\section{B. Local-density approximation calculations}

To gain further insight into the stability and structure of the predicted structure, first-principles total-energy calcula- 
TABLE I. Distribution of Si and O coordination and phases produced for different $\sigma_{i j}$ from the molecular-dynamics simulations at 300 $\mathrm{K}$. The runs designated with a $C$ are compression runs, while those with a $D$ correspond to decompression runs from the specified stresses to ambient conditions (zero stress).

\begin{tabular}{|c|c|c|c|c|c|c|}
\hline$\left(\sigma_{x x}, \sigma_{y y}, \sigma_{z z}\right), \mathrm{GPa}$ & ${ }^{\mathrm{IV}} \mathrm{Si}$ & ${ }^{\mathrm{v}_{\mathrm{Si}}}$ & ${ }^{\mathrm{VI}} \mathrm{Si}$ & ${ }^{\mathrm{II}} \mathrm{O}$ & ${ }^{\mathrm{III}} \mathrm{O}$ & Recovered phase \\
\hline$(0,0,0)$ & $100 \%$ & $0 \%$ & $0 \%$ & $100 \%$ & $0 \%$ & $\alpha$-quartz \\
\hline$(22,22,22) C$ & $12.5 \%$ & $42 \%$ & $45.5 \%$ & $34.5 \%$ & $65.5 \%$ & Amorphous \\
\hline$(22,22,22) D$ & $75 \%$ & $23.5 \%$ & $1.5 \%$ & $87 \%$ & $13 \%$ & Amorphous \\
\hline$(25,25,25) C$ & $8 \%$ & $38.5 \%$ & $53.5 \%$ & $30 \%$ & $70 \%$ & Amorphous \\
\hline$(25,25,25) D$ & $69 \%$ & $26 \%$ & $5 \%$ & $82 \%$ & $18 \%$ & Amorphous \\
\hline$(30,30,30) C$ & $7 \%$ & $36.5 \%$ & $56.5 \%$ & $28 \%$ & $72 \%$ & Amorphous \\
\hline$(30,30,30) D$ & $56 \%$ & $36 \%$ & $8 \%$ & $73 \%$ & $27 \%$ & Amorphous \\
\hline$(22,22,13) C$ & $13 \%$ & $34 \%$ & $53 \%$ & $32 \%$ & $68 \%$ & Amorphous \\
\hline$(22,22,13) D$ & $97 \%$ & $3 \%$ & $0 \%$ & $98 \%$ & $2 \%$ & Defective $\alpha$-quartz \\
\hline$(20,20,27) C$ & $0 \%$ & $100 \%$ & $0 \%$ & $50 \%$ & $50 \%$ & Penta \\
\hline$(20,20,27) D$ & $100 \%$ & $0 \%$ & $0 \%$ & $100 \%$ & $0 \%$ & $\alpha$-quartz \\
\hline$(20,20,29) C$ & $0 \%$ & $90 \%$ & $10 \%$ & $45 \%$ & $55 \%$ & Penta + amorphous \\
\hline$(20,20,29) D$ & $99 \%$ & $1 \%$ & $0 \%$ & $99 \%$ & $1 \%$ & Defective $\alpha$-quartz \\
\hline
\end{tabular}

tions were undertaken using a density-functional framework $^{48}$ within the local-density approximation (LDA) to electron exchange and correlation. A preconditioned conjugate-gradient method was used to minimize the electronic degrees of freedom. ${ }^{49}$ The electronic wave functions were expanded in a plane-wave basis set with periodic boundary conditions. Norm-conserving pseudopotentials constructed within the scheme developed by Troullier and Martins $^{50}$ were used. The oxygen potential was generated using neutral $2 s^{2} 2 p^{4}$ as the reference state with a radial cutoff of 1.45 bohr for both the $s$ and $p$ components of the potential, and the radius of the nonlinear core correction was 0.57 bohr. $^{51}$ The silicon potential was generated using $3 s^{2} 3 p^{2} 3 d^{0}$ as a reference state with a radial cutoff of 2.09 bohr for the $s, p$, and $d$ components of the potential. The $s$ and $p$ angular momentum channels were treated as nonlocal components of the potential each with two radial projection functions, and the $d$ channel was treated as the local component of the potential. The radius of the nonlinear core correction was taken to be 1.81 bohr. Calculations performed at various kinetic energy cutoffs indicated that a cutoff of 30 hartrees was required for satisfactory convergence of the structural parameters. The number of special $k$ points for the Brillouin-zone integration was increased until the total energies had converged to below $1 \mathrm{~m}$ hartree/atom. For the penta structure, we used a [442] Monkhorst-Pack grid ${ }^{52}$ giving five special points in the irreducible Brillouin zone.

\section{RESULTS}

\section{A. Crystal structure}

We begin with the results of molecular-dynamics calculations. When hydrostatic stresses are applied on the MD box containing crystalline quartz, pressure-induced amorphization occurs at $22 \mathrm{GPa},{ }^{27}$ in good agreement with other simulations (e.g., Refs. 53 and 54). However, different behavior is observed when the simulations are performed in the presence of deviatoric stresses (nonhydrostatic pressure). There is a tendency to form a different crystalline phase; for a stress of $27 \mathrm{GPa}$ along the $c$ axis and $20 \mathrm{GPa}$ in the $a, b$ plane the crystallization is complete. The principal results of the simulations are summarized in Table I. In order to further characterize the structure of the phase, a reference state was first created by hydrostatic compression of quartz up to $20 \mathrm{GPa}$. This compression was carried out over 134 ps integration time. All systems were allowed to age for $13.4 \mathrm{ps}$ in order to insure that the structure was well relaxed. The system was then uniaxially compressed (67 ps integration time) along the $c$ axis up to the phase transition at $27 \mathrm{GPa}$ and also relaxed during 13.4 ps. The resulting structure of the phase is shown in Fig. 1.

The cell parameters, atomic positions, and symmetry elements were obtained as follows. A theoretical x-raydiffraction pattern was constructed from the coordinates of the 480 silicon and 960 oxygen atoms given in the Cartesian system defined by the orthorhombic MD box. ${ }^{55}$ The $d$ 's from the 14 strongest peaks were selected and indexed. ${ }^{56}$ The cell parameters were then refined from the full set of observable

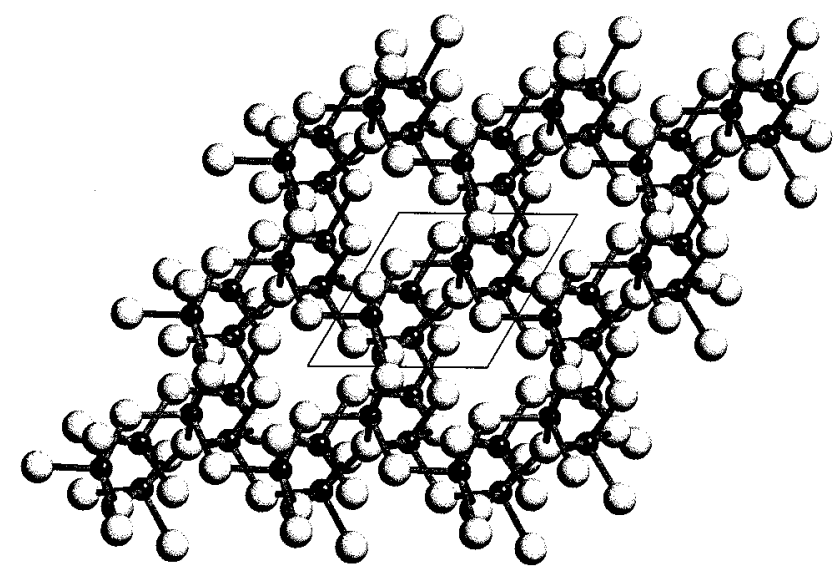

FIG. 1. The high-pressure phase viewed down the $c$ axis. The small (dark) spheres and large (white) spheres are the silicon and oxygen atoms, respectively. All silicons are linked to five oxygens, whereas half the oxygens are linked to two silicons, the other half being linked to three silicon atoms. The unit cell is shown in the center. 


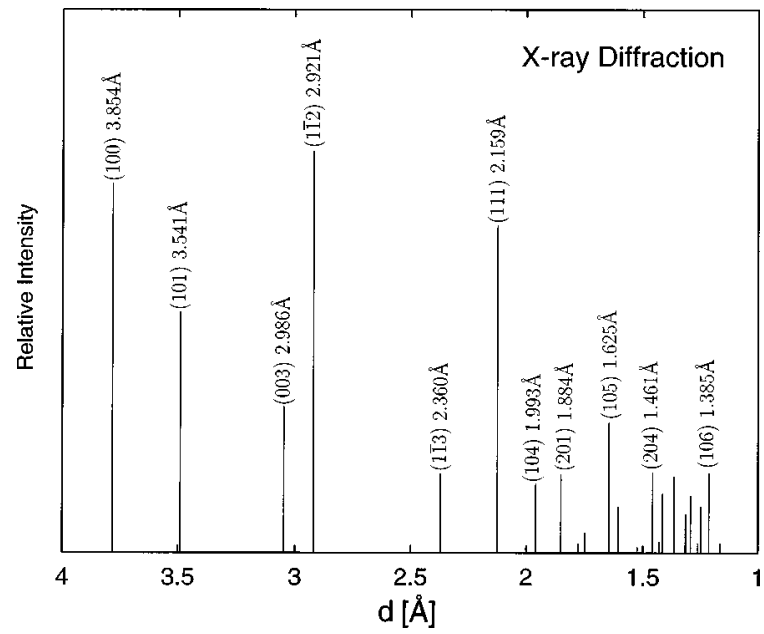

FIG. 2. Calculated powder x-ray-diffraction pattern of the $\mathrm{SiO}_{2}$ penta for the relaxed structure $(P=16 \mathrm{GPa})$.

peak positions. The position and orientation of the unit cell within the orthorhombic box was then determined by choosing the centermost $\mathrm{Si}$ atom as the origin and finding the other $\mathrm{Si}$ atoms that were related to it by translational lattice symmetry, with the unit-cell distances thus defining a transformation matrix from the Cartesian system of the orthorhombic box to the direct crystal space. All the atoms were then projected into the unit cell. The latter contained 18 atoms and this produced a distribution of 80 atoms at each of the sites. The position for each atom in the unit cell was chosen to be the mean of this distribution. The positions of the atoms in the unit cell were then analyzed to determine its possible symmetry operations. ${ }^{57}$ Prototype asymmetric unit positions were arbitrarily chosen and each atom was then mapped onto its corresponding prototype, producing three distributions of six atoms each. The average of these distributions gave the positions assigned to the asymmetric unit. The $d$ spacings of the 14 most intense peaks were indexed with trigonal symmetry. Every peak could then be indexed under this symmetry with a very high figure of merit of $214 .{ }^{58}$ The space group was found to be $P 3_{2} 21$ (No.154) within a tolerance of $0.0038 \AA$. This is identical to the space group of $\alpha$-quartz.

The cell parameters were then refined by energy minimization with both the interatomic potential and LDA methods. The same space group was found when the structure was relaxed under $P 1$ symmetry with both methods, and the structural parameters are in good agreement. The resulting powder x-ray-diffraction pattern for the optimized structure is shown in Fig. 2.

\section{B. Local structure and coordination}

The structure consists of silicon in fivefold coordination by oxygen, with oxygen atoms in both two- and threefold coordination with silicon. The $\mathrm{SiO}_{5}$ pentahedra form a distorted square pyramid linked together as edge-sharing dimers to form a three-dimensional network. A detail of the distorted square pyramidal pentahedron is shown in Fig. 3, and the structural parameters at a reference hydrostatic pressure of $16 \mathrm{GPa}$ are given in Table II. The mean Si-O distance within a pentahedron $(1.703 \AA$ from LDA; $1.680 \AA$ from BKS at this pressure) falls between that typically observed in

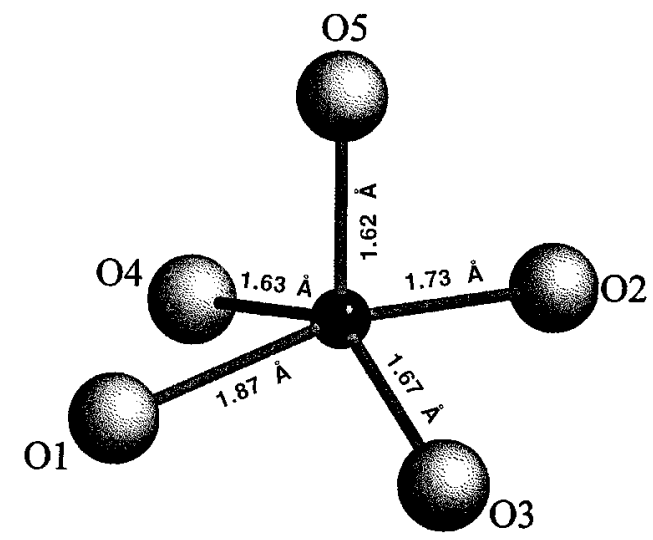

FIG. 3. Detail of the $\mathrm{SiO}_{5}$ polyhedron showing the labeling of the atoms and bond lengths calculated by LDA ( $P=16 \mathrm{GPa})$.

$\mathrm{SiO}_{4}(1.61 \AA)$ and $\mathrm{SiO}_{6}$ polyhedra $(1.79 \AA)^{3}{ }^{3}$ According to the LDA result, four of the five Si-O bond lengths within the $\mathrm{SiO}_{5}$ units range from 1.617 to $1.728 \AA$, whereas the bond between the $\mathrm{Si}$ and the apical $\mathrm{O}$ is longer value of $1.869 \AA$. This result is also reflected in the $\mathrm{Si}-\mathrm{O}$ radial distribution function (RDF), which is compared to those calculated for $\alpha$-quartz and stishovite in Fig. 4. These were computed from the simulations with the BKS potential by averaging over 10000 configurations, and the local atomic coordination number was determined by integrating all atomic distances lower than the first minimum in the RDF. The large anisotropy in bond lengths, which is not observed in $\mathrm{SiO}_{4}$ or $\mathrm{SiO}_{6}$ polyhedra, seems to be characteristic of the $\mathrm{SiO}_{5}$ units. Also, two O-Si-O angles are close to $90^{\circ}\left(97\right.$ and $94^{\circ}$ ) while the two others are larger: 104 and $112^{\circ}$. Notably, the calculated distortion of the pentahedra is similar to that recently

TABLE II. Structural parameters of penta-SiO ${ }_{2}(P=16 \mathrm{GPa})$ (space group $P 3_{2} 21$ ).

\begin{tabular}{|c|c|c|}
\hline & LDA & BKS Potential \\
\hline$a$ & $4.414 \AA$ & $4.462 \AA$ \\
\hline$c$ & $9.358 \AA$ & $9.123 \AA$ \\
\hline $\operatorname{Si}(x, y, z)$ & $\begin{array}{llll}0.5126 & 0.3224 & 0.2204\end{array}$ & $\begin{array}{lll}0.5045 & 0.3087 & 0.2214\end{array}$ \\
\hline $\mathrm{O}(1)(x, y, z)$ & $\begin{array}{llll}0.4839 & 0.0761 & 0.0537\end{array}$ & $\begin{array}{lll}0.4776 & 0.0775 & 0.0526\end{array}$ \\
\hline $\mathrm{O}(2)(x, y, z)$ & $\begin{array}{lll}0.8253 & 0.6686 & 0.1439\end{array}$ & $\begin{array}{llll}0.8200 & 0.6431 & 0.1425\end{array}$ \\
\hline $\mathrm{Si}-\mathrm{O}(1)$ & $1.869 \AA$ & $1.824 \AA$ \\
\hline $\mathrm{Si}-\mathrm{O}(2)$ & $1.728 \AA$ & $1.714 \AA$ \\
\hline $\mathrm{Si}-\mathrm{O}(3)$ & $1.674 \AA$ & $1.636 \AA$ \\
\hline $\mathrm{Si}-\mathrm{O}(4)$ & $1.626 \AA$ & $1.620 \AA$ \\
\hline $\mathrm{Si}-\mathrm{O}(5)$ & $1.617 \AA$ & $1.605 \AA$ \\
\hline $\mathrm{O}(1)-\mathrm{Si}-\mathrm{O}(2)$ & $168.2^{\circ}$ & $169.2^{\circ}$ \\
\hline $\mathrm{O}(1)-\mathrm{Si}-\mathrm{O}(3)$ & 76.3 & 76.5 \\
\hline $\mathrm{O}(1)-\mathrm{Si}-\mathrm{O}(4)$ & 87.6 & 85.8 \\
\hline $\mathrm{O}(1)-\mathrm{Si}-\mathrm{O}(5)$ & 94.6 & 94.6 \\
\hline $\mathrm{O}(2)-\mathrm{Si}-\mathrm{O}(3)$ & 95.9 & 96.1 \\
\hline $\mathrm{O}(2)-\mathrm{Si}-\mathrm{O}(4)$ & 93.7 & 91.8 \\
\hline $\mathrm{O}(2)-\mathrm{Si}-\mathrm{O}(5)$ & 96.3 & 99.1 \\
\hline $\mathrm{O}(3)-\mathrm{Si}-\mathrm{O}(4)$ & 142.4 & 140.8 \\
\hline $\mathrm{O}(3)-\mathrm{Si}-\mathrm{O}(5)$ & 108.9 & 112.7 \\
\hline $\mathrm{O}(4)-\mathrm{Si}-\mathrm{O}(5)$ & 106.1 & 103.7 \\
\hline
\end{tabular}




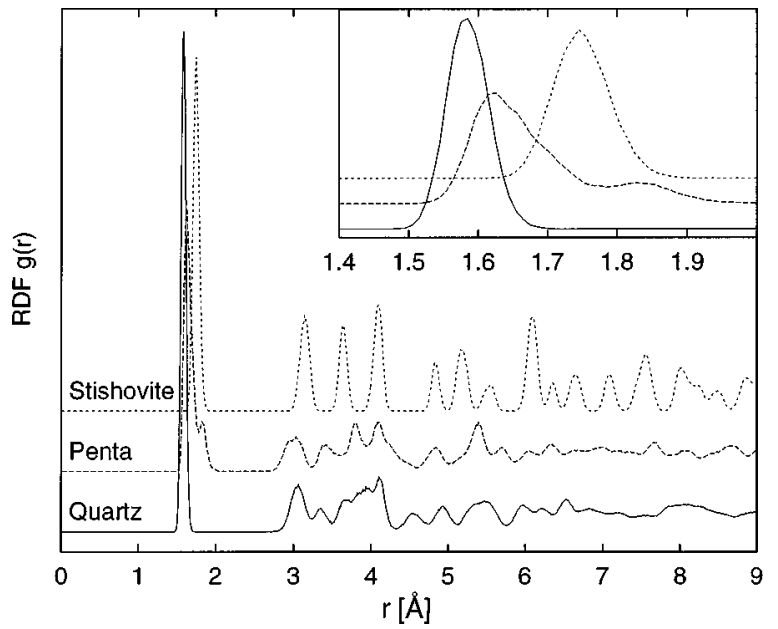

FIG. 4. Radial distribution function (RDF) for silicon and oxygen $(16 \mathrm{GPa}$ and $300 \mathrm{~K})$. The reported first-neighbor distances for penta- $\mathrm{SiO}_{2}$ lie between that for quartz and stishovite. The computed average $\mathrm{Si}-\mathrm{O}$ bond lengths are 1.60, 1.68, and $1.75 \AA$, for $\alpha$-quartz, penta- $\mathrm{SiO}_{2}$, and stishovite, respectively.

observed for a calcium silicate phase consisting of ${ }^{{ }^{V}} \mathrm{Si}^{59,60}$ The Si-O bond lengths determined in that study (measured at zero pressure) are $1.671,1.682,1.696,1.781$, and $1.824 \AA$ with an average value of $1.731 \AA$.

\section{Band structure}

The electronic band structures for both penta and $\alpha$-quartz were also calculated. Figure 5 shows the band structures and associated total density of states (TDOS) for the two phases along high-symmetry directions of the Brillouin zone of the hexagonal unit cell at a common pressure of $16 \mathrm{GPa}$. The zero of the energy scale corresponds to the valence band maximum. The penta phase is an insulator with a direct gap calculated by LDA of $4.9 \mathrm{eV}$ at $\Gamma$, whereas $\alpha$-quartz has an indirect gap calculated to be $6.4 \mathrm{eV}$ (valence band maximum at $K$ and a conduction band minimum at $\Gamma$ ).

At ambient pressure, the valence-band structure of $\alpha$-quartz is separated into three distinct regions (for a review, see Ref. 42). The lowest is composed mainly of O $2 s$ states with a small contribution from $\mathrm{Si} 3 s$ and $3 p$ states. The middle is composed of $\mathrm{O} 2 p$ and $\mathrm{Si} 3 s$ and $3 p$ states with a small contribution from the $\mathrm{Si} 3 d$ states. The states in this region contribute principally to the $\mathrm{Si}-\mathrm{O}$ bonding. The top of the valence band is primarily composed of $\mathrm{O} 2 p$ nonbonding states, with a very small contribution from $\mathrm{Si} 3 p$ and $3 d$. At $16 \mathrm{GPa}$ however, the middle (bonding) and top (nonbonding) regions have merged [Figs. 5(b) and 5(d)]. This result is in agreement with the previous work on $\alpha$-quartz by Binggeli et $a l .{ }^{61}$ and Di Pomponio and Continenza. ${ }^{62}$ It has been suggested that this rehybridization can favor $\mathrm{Si}-\mathrm{O}$ bonding with the silicon in higher coordination. ${ }^{61,62}$ The band structure and TDOS [Figs. 5(a) and 5(c)] of penta-SiO $\mathrm{S}_{2}$ reveal significantly more mixing of these regions than occurs in $\alpha$-quartz, but less than that found for stishovite. ${ }^{63}$

\section{Vibrational density of states}

The vibrational densities of states (VDOS) of the penta phase is of interest because it contains the signature of the
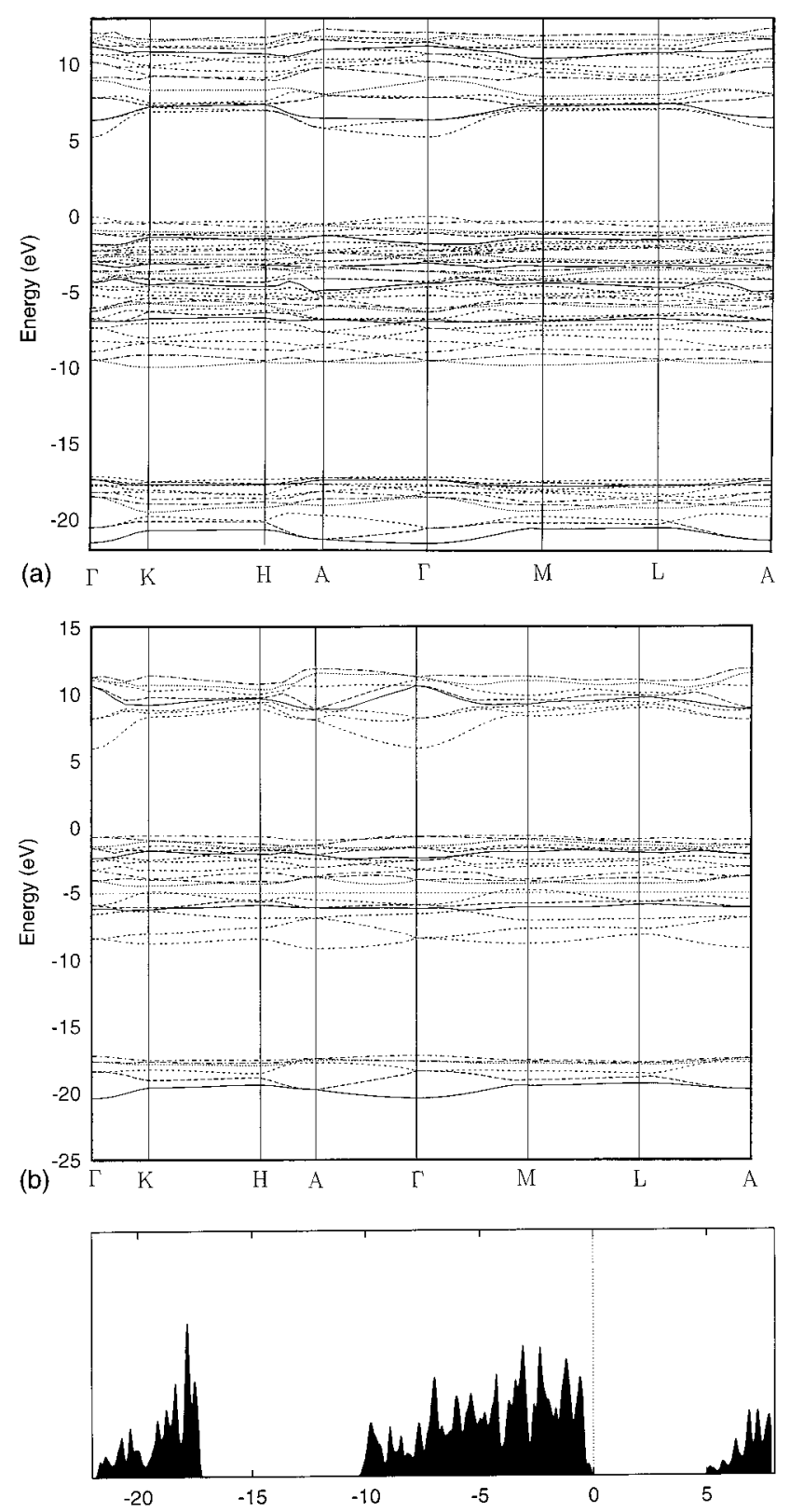

(c)

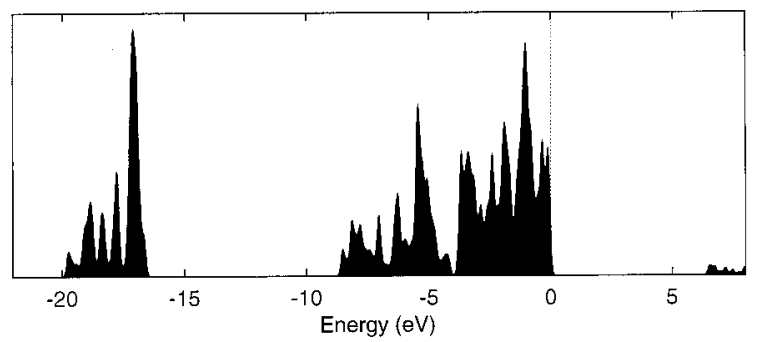

FIG. 5. Band structures: (a) penta-SiO ${ }_{2}$, (b) $\alpha$-quartz; total density of states: (c) penta- $\mathrm{SiO}_{2}$, (d) $\alpha$-quartz. The calculations were performed at a common pressure of $16 \mathrm{GPa}$.

vibrations related to $\mathrm{SiO}_{5}$ units which could serve to identify these units by neutron inelastic-scattering, infrared, or Raman spectroscopy. The VDOS of quartz and penta- $\mathrm{SiO}_{2}$ were computed by MD at $16 \mathrm{GPa}$ (see Fig. 6). The VDOS were calculated from the Fourier transform of the mass weighted 


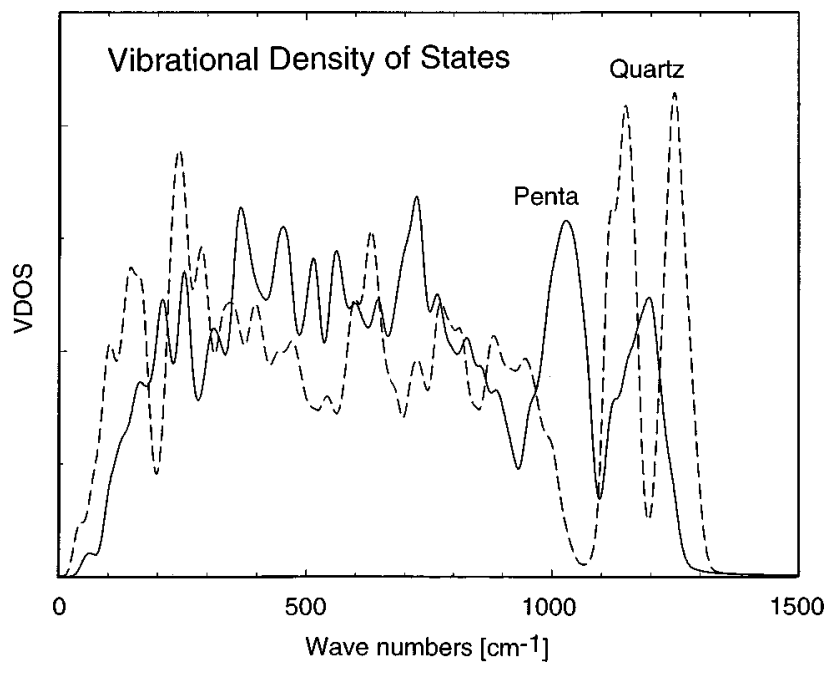

FIG. 6. Vibrational density of state of the penta phase and $\alpha$-quartz calculated using the power spectrum of the velocity autocorrelation function $(P=16 \mathrm{GPa} ; T=300 \mathrm{~K})$.

power spectrum of the velocity autocorrelation functions (VAF's), ${ }^{64}$ computed in the $(N, V, E)$ microcanonical ensemble. The VAF's were calculated for runs up to $2.68 \mathrm{ps}$ and averaged over 2000 configurations after a 13.4 ps constant energy run. They have been smoothed by a Gaussian distribution of width $10 \mathrm{~cm}^{-1}$ to be comparable to the resolution of typical inelastic neutron-scattering experiments. There are a number of important differences. A clear gap in the VDOS of quartz exists between the lower frequency modes (up to $900 \mathrm{~cm}^{-1}$ ) and those at $1100-1350 \mathrm{~cm}^{-1}$. This gap is present in the measured Raman and IR spectra of quartz (and other $\mathrm{SiO}_{2}$ polymorphs consisting of $\mathrm{SiO}_{4}$ units), ${ }^{65}$ but it is less pronounced in the VDOS predicted for the new phase. The higher frequency group of modes corresponds to asymmetrical $\mathrm{Si}-\mathrm{O}$ stretching motions within the $\mathrm{SiO}_{4}$ units. The corresponding modes in the penta phase involve similar vibrations of $\mathrm{SiO}_{5}$ groups, but they are shifted toward lower wave numbers $\left(950-1250 \mathrm{~cm}^{-1}\right)$. The lower wave numbers reflects the longer mean $\mathrm{Si}-\mathrm{O}$ bond length, similar to the downward frequency shift and longer bond lengths documented for the $\mathrm{SiO}_{6}$ octahedra (e.g., in stishovite $\left.{ }^{4,16}\right)$. The broadening of this group of modes in the penta phase results from the existence of different bond lengths within the $\mathrm{SiO}_{5}$ groups in comparison to the $\mathrm{SiO}_{4}$ tetrahedra of quartz. This difference in VDOS between quartz and penta- $\mathrm{SiO}_{2}$ thus should serve as a signature of the fivefold coordination of $\mathrm{Si}$, because these features of the VDOS should be reflected in the Raman and IR spectra. ${ }^{65}$ Differences between quartz and the penta phase are also observed below $900 \mathrm{~cm}^{-1}$, but they are less diagnostic than those observed at higher wave numbers.

\section{E. Stability and equation of state}

A series of calculations were performed to examine the stability of the phases as a function of compression and decompression. The LDA calculations confirm the transition from quartz to the penta phase obtained in the MD simulations. The energy-volume curves calculated by LDA for the

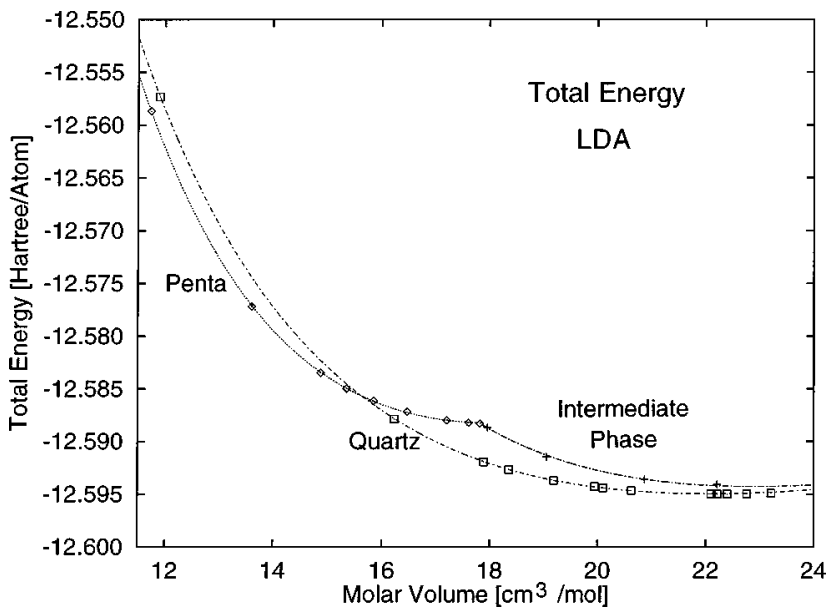

FIG. 7. Energy as a function of volume calculated by LDA. The structure is fully optimized at each volume.

phases are shown in Fig. 7, where the calculated points are fit to a Birch-Murnaghan equation of state. ${ }^{66}$ The penta phase is metastable with respect to quartz above a critical volume of $15.78 \mathrm{~cm}^{3} /$ mole $\left(3.80 \mathrm{~g} / \mathrm{cm}^{3}\right)$. This result is in agreement with the MD calculations which show that the quartz-topenta transition takes place when quartz reaches a critical volume of $17.29 \mathrm{~cm}^{3} / \mathrm{mol}\left(3.47 \mathrm{~g} / \mathrm{cm}^{3}\right)$. The molar volume of the penta phase obtained by MD $\left(15.67 \mathrm{~cm}^{3} / \mathrm{mol}\right)$ at the transition pressure is very close to that obtained by LDA.

The $P-V$ equations of state calculated from the energyvolume curves are shown in Fig. 8. The results are close to those determined from the BKS potential. We also compare both the LDA and BKS results for quartz and stishovite, as well as experimental results for both phases. ${ }^{67-74}$ Both the molar volume and compressibility of penta- $\mathrm{SiO}_{2}$ are intermediate between those of $\alpha$-quartz and stishovite at all pressures. The good agreement between theory and experiment for quartz and stishovite, also shown in previous studies using both LDA (Ref. 75) and the BKS potential, ${ }^{53,76}$ supports the reliability of the prediction for the metastable phase.

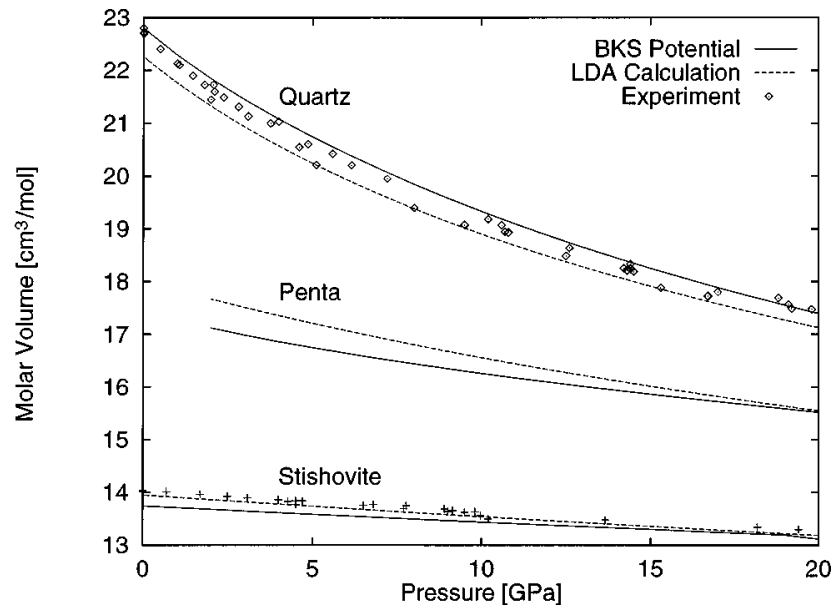

FIG. 8. The pressure-volume equation of state for penta- $\mathrm{SiO}_{2}$, $\alpha$-quartz, and stishovite. The solid and dashed lines are the LDA and the interatomic potential results (static lattice). The symbols are experimental room-temperature static compression data for $\alpha$-quartz (Refs. 67-71) and stishovite (Refs. 72-74). 


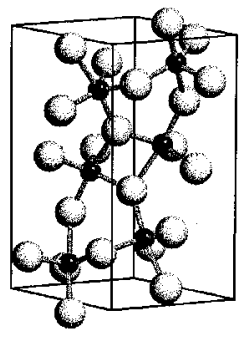

a

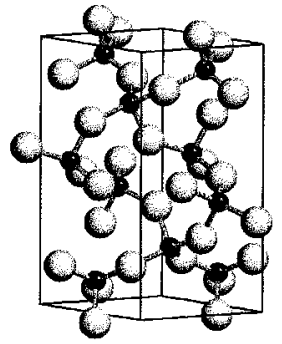

b

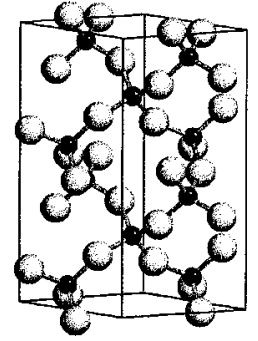

c
FIG. 9. Comparison of the crystal structures of (a) penta- $\mathrm{SiO}_{2}$, (b) the intermediate phase, and (b) $\alpha$-quartz. The atoms are numbered in Fig. 3.

\section{F. Phase transformations}

Both the MD and LDA calculations provide insights on the transitions on compression or decompression. MD simulations at the pressure of formation predict that the penta$\mathrm{SiO}_{2}$ withstands temperatures up to $1500 \mathrm{~K}$ without exhibiting any phase transitions. Upon further uniaxial pressurization at room temperature, the penta phase undergoes partial amorphization for a stress along the $c$ direction $2 \mathrm{GPa}$ above that at which it is formed. In contrast with the solid-state amorphization of quartz, which yields a mixture of ${ }^{\mathrm{IV}} \mathrm{Si}$,

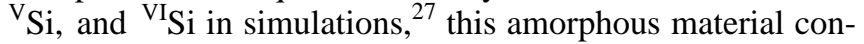
tains only silicon atoms in five- and sixfold coordination. When decompressed, this amorphous phase undergoes a partially reversible transition to a defective quartzlike material at $10 \mathrm{GPa}$ (Table I).

Both the LDA and BKS calculations indicate that the penta phase is energetically stable with respect to quartz below a critical volume. MD simulations carried out on decompression predict that penta- $\mathrm{SiO}_{2}$ reverts to $\alpha$-quartz at a pressure of $2 \mathrm{GPa}$ when the decompression path is identical to that followed on compression (i.e., the excess uniaxial stress is first released from $27 \mathrm{GPa}$ down to $20 \mathrm{GPa}$ and the hydrostatic environment is maintained during further decompression). Both the LDA calculations and molecular mechanics methods show that penta- $\mathrm{SiO}_{2}$ transforms to a transient intermediate structure before fully relaxing to $\alpha$-quartz. This intermediate phase, which also has ${ }^{\mathrm{IV}} \mathrm{Si}$, is therefore an unstable phase in the system. The structures are compared in Fig. 9.

The mechanism of the transformation was examined in detail. Because of the bond breaking along the transformation path and the existence of $\mathrm{Si}-\mathrm{O}$ bonds outside the range of parametrization of the BKS potential, the LDA results are considered more reliable. The results show that during decompression the $\mathrm{SiO}_{5}$ polyhedra first expand and distort, as shown in Fig. 10. Both the Si-O bond lengths and $\mathrm{Si}-\mathrm{O}-\mathrm{Si}$ angles increase for the two-coordinated oxygens. The transformation to the intermediate structure occurs when the Si-O bond of the apical oxygen breaks (a distance of approximately $2.0 \AA$ ). At this point, the four remaining $\mathrm{Si}-\mathrm{O}$ distances rapidly decrease, transforming the $\mathrm{SiO}_{5}$ pentahedra into distorted $\mathrm{SiO}_{4}$ tetrahedra. These tetrahedra then rotate to form the $\alpha$-quartz structure to reduce the strain.

\section{DISCUSSION}

Despite the large number of high-pressure studies of quartz in recent years, the effects of nonhydrostatic stresses

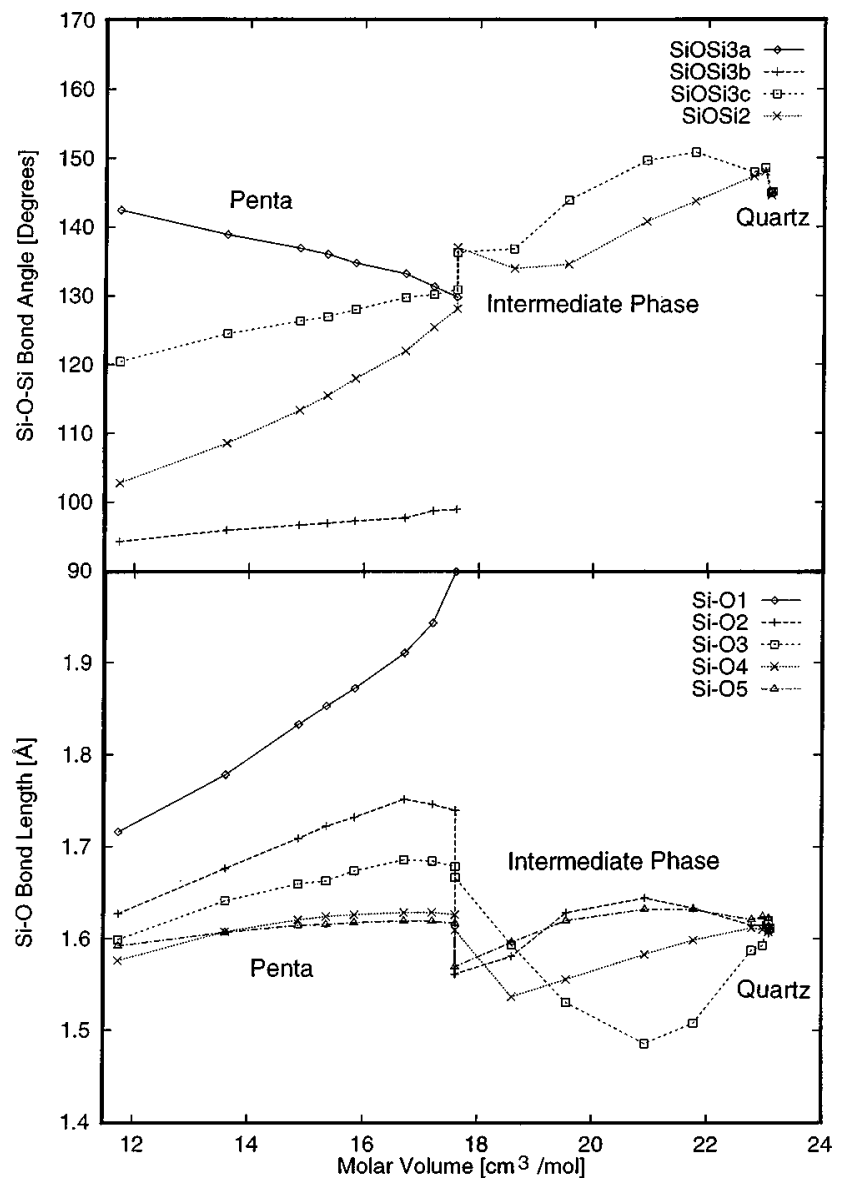

FIG. 10. Structural variations in the penta phase as a function of volume during decompression. Si-O-Si bond angles (top). Si-O bond distances (bottom).

on its phase transitions, structures, and dynamics, are not fully understood. The present calculations indicate that specific nonhydrostatic stress conditions can radically alter the compression behavior of $\alpha$-quartz. Lattice-dynamics calculations performed with the BKS potential predict a pressureinduced phonon softening in hydrostatically compressed quartz; complete softening is calculated to occur at $21 \mathrm{GPa}$, prior to the onset of a mechanical instability. ${ }^{77}$ Another study using lattice dynamics calculations has predicted a dynamical instability at $18.5 \mathrm{GPa}$ in the $[1 / 3,1 / 3,0]$ direction of the Brillouin zone. ${ }^{78}$ This transition pressure is close to that of the experimentally reported crystalline-crystalline phase transition documented in high-pressure hydrostatic experiments of quartz. ${ }^{7}$ The present study strongly suggests that when quartz is compressed in a nonhydrostatic manner with the stress along $c$ greater than that along $a$ and $b$, this instability is postponed to higher pressures. The resulting strain allows the silicon atoms to experience a different oxygen environment than would normally be encountered under hydrostatic conditions. In the case of a stress tensor with $\sigma_{x x}$ $=20 \mathrm{GPa}, \sigma_{y y}=20 \mathrm{GPa}, \sigma_{z z}=27 \mathrm{GPa}$, a low-energy pathway is created between quartz to the penta structure, thereby facilitating the transformation to the penta phase.

As discussed above, the ${ }^{\mathrm{V}_{\mathrm{Si}}} \mathrm{Si}$ phase is produced by application of deviatoric stresses but is stable mechanically and energetically (e.g., with respect to quartz) at fixed hydrostatic 
pressure. An unusual sequence of transformations occurs as a function of decreasing hydrostatic pressure. There appears to be a continuous, low-energy path between the penta, the intermediate phase, and $\alpha$-quartz transition. During the transformation, the coordination number of the silicon changes from five to four, and several of the oxygens change from three- to twofold. Notably, this takes place with no change in $P 3_{2} 21$ symmetry, even when the symmetry constraints are relaxed (i.e., calculations under $P 1$ symmetry). Christy ${ }^{79}$ has denoted this class of phase transformations, in which no change of space group occurs, as type 0 . Such isosymmetric phase transitions have been shown to occur in feldspars, pyroxenes, ${ }^{80}$ and nonlinear optical materials (e.g., $\left.\mathrm{KTiPO}_{4}\right)^{81}$

As the two phases identified here have not been observed experimentally, it is useful to examine the accuracy of the theoretical techniques used in the calculations. Numerous studies have tested the reliability of the BKS potential, which is similar to the earlier potential of Tsuneyuki et al. ${ }^{82}$ but is more accurate for a number of physical properties of crystalline polymorphs (e.g., Ref. 76). As illustrated in Fig. 8, both the LDA and interatomic potential calculations are able to accurately reproduce the experimental compression behavior of both $\alpha$-quartz ${ }^{67-71}$ and stishovite. ${ }^{72-74}$ Despite the success of the model potential, such methods are considered inherently approximate, and indeed problematic for processes such as bond breaking and making, so it is useful to consider more accurate methods. We believe that the LDA calculations are more reliable for predicting the structural properties of penta- $\mathrm{SiO}_{2}$ as well as the decompression behavior. It has been reported recently that generalized gradient approximations (GGA) to the exchange and correlation energy are required to describe accurately the energy difference between $\alpha$-quartz and stishovite. ${ }^{83}$ While the GGA gives a more correct energy difference, it appears to do so at the expense of accuracy of the structural parameters. Our interest in the structural parameters of the five-coordinated silica phase therefore justifies the use of the LDA. Additional studies using the GGA and other extensions of LDA would be valuable.

It is useful to examine measurable signatures of the structure such as the diffraction pattern and vibrational properties to aid in the possible experimental identification of the penta phase. This is of interest because of the possibility of whether such material may have been formed in small quantities during previous high-pressure experiments. Studies of metastable transitions in silica, and quartz in particular, produce heterogeneous samples, with small amounts of crystalline (or partially crystalline material) mixed with material having disorder ranging from extensive twinning to com- pletely amorphous. ${ }^{6,7}$ Both vibrational and diffraction measurements yield broadbands. Deviatoric stresses are present to some degree in most experimental studies: Under such conditions, local stresses within the heterogeneous sample are expected to be significant, even if the sample assemblage is in a hydrostatic environment, because of local grain-grain-contacts. ${ }^{3} \mathrm{We}$ anticipate that the calculated VDOS may be useful for spectroscopic identification of either the penta phase or the presence of $\mathrm{SiO}_{5}$ units in crystalline or amorphous silicates. It may useful to compare both predicted diffraction peaks and vibrational properties for penta- $\mathrm{SiO}_{2}$ with existing and future measurements on such compressed metastable silica samples. As discussed above, a high-pressure phase of $\mathrm{CaSi}_{2} \mathrm{O}_{5}$ consisting of ${ }^{\mathrm{V}} \mathrm{Si}$ has recently been synthesized and characterized. ${ }^{59,60}$ The silicon and oxygen form distorted square pyramidal pentahedra, and the structural parameters, including the $\mathrm{Si}-\mathrm{O}$ bond lengths, are similar to those predicted for the pure $\mathrm{SiO}_{2}$ phase.

In conclusion, this study shows that it is possible to control transformation pathways of quartz to new polymorphs, and as such, it illustrates the continued richness of the $\mathrm{SiO}_{2}$ system. It appears possible to use such methods to tune pathways as functions of hydrostatic and nonhydrostatic stress to find phases in other chemical systems. While variable stress has long been used in the synthesis of thin films through the control of lattice mismatch between the substrate and film, we suggest it is possible to synthesize new materials by using deviatoric stress to control the pathway of the phase transformation. This is of added interest in view of recently developed experimental techniques for controlling and directly measuring the three-dimensional distribution of stress and strain in samples under high confining pressures (e.g., in diamond-anvil cells) ${ }^{84,85}$ Such techniques may allow synthesis of crystalline phases that cannot be obtained by application of hydrostatic pressure, and therefore could be important for solid-state engineering and creation of new materials.

\section{ACKNOWLEDGMENTS}

We are grateful to C. T. Prewitt and R. E. Cohen for comments on the manuscript and useful discussions. We thank the Pôle Scientifique de Modélisation Numérique (ENS-Lyon), the Cornell Materials Science Computing Center, and the Virginia Tech Computing Center for use of CONVEX SPP 1000, IBM RS/6000 and SP2 computing resources, respectively. D.M.T. thanks Doug Allan of Corning, Inc. for valuable contributions. This work was partially supported by the NSF. The Center for High Pressure Research is an NSF Science and Technology Center.
*Present address: Department of Geosciences, University of Arizona, Tucson, AZ 85721

${ }^{1}$ Silica-Physical Behavior, Geochemistry, and Materials Applications, Reviews of Mineralogy, edited by P. J. Heaney, G. V. Gibbs, and C. T. Prewitt (Mineral. Soc. America, Washington, DC, 1994), Vol. 29.

${ }^{2}$ R. B. Sosman, The Phases of Silica (Rutgers University Press, New Brunswick, NJ, 1965)

${ }^{3}$ R. J. Hemley, C. T. Prewitt, and K. J. Kingma, Silica-Physical
Behavior, Geochemistry, and Materials Applications, Reviews of Mineralogy (Ref. 1), p. 41.

${ }^{4}$ R. J. Hemley, High-Pressure Research in Mineral Physics, edited by M. H. Manghnani and Y. Syono (Terra Scientific-Tokyo, AGU-Washington, DC, 1987), p. 347.

${ }^{5}$ R. J. Hemley, A. P. Jephcoat, H. K. Mao, L. C. Ming, and M. H. Manghnani, Nature (London) 334, 52 (1988).

${ }^{6}$ K. J. Kingma, C. Meade, R. J. Hemley, H. K. Mao, and D. R. Veblen, Science 259, 666 (1993). 
${ }^{7}$ K. J. Kingma, R. J. Hemley, H. K. Mao, and D. R. Veblen, Phys. Rev. Lett. 70, 3927 (1993).

${ }^{8}$ J. P. Itié, A. Polian, G. Calas, J. Petiau, A. Fontaine, and H. Tolentino, High Press. Res. 5, 717 (1990).

${ }^{9}$ M. Kruger and R. Jeanloz, Science 249, 647 (1990).

${ }^{10}$ M. Madon, P. Gillet, and B. Reynard, Geochim. Cosmochim. Acta 58, 3653 (1994).

${ }^{11}$ P. Gillet, J. Badro, B. Varel, and P. McMillan, Phys. Rev. B 51, 11262 (1995).

${ }^{12}$ K. J. Kingma, R. E. Cohen, R. J. Hemley, and H. K. Mao, Nature (London) 374, 243 (1995).

${ }^{13}$ R. J. Hemley, H. K. Mao, P. M. Bell, and B. O. Mysen, Phys. Rev. Lett. 57, 747 (1986).

${ }^{14}$ Q. Williams and R. Jeanloz, Science 239, 902 (1988).

${ }^{15}$ C. Meade, R. J. Hemley, and H. K. Mao, Phys. Rev. Lett. 69, 1387 (1992).

${ }^{16}$ Q. Williams, R. J. Hemley, M. B. Kruger, and R. Jeanloz, J. Geophys. Res. 98, 22157 (1993).

${ }^{17}$ C. A. Angell, P. A. Cheeseman, and S. Tammadon, Science 218, 885 (1982).

${ }^{18}$ J. D. Kubicki and A. C. Lasaga, Am. Mineral. 73, 941 (1988).

${ }^{19}$ J. D. Kubicki and A. C. Lasaga, Phys. Chem. Miner. 17, 661 (1991).

${ }^{20}$ J. R. Rustad, D. A. Yuen, and F. J. Spera, J. Geophys. Res. 96, 19965 (1991).

${ }^{21}$ J. S. Tse, D. D. Klug, and Y. L. Page, Phys. Rev. B 46, 5933 (1992).

${ }^{22}$ J. R. Rustad, D. A. Yuen, and F. J. Spera, Chem. Geol. 96, 421 (1992).

${ }^{23}$ B. T. Poe, P. F. McMillan, C. A. Angell, and R. K. Sato, Chem. Geol. 96, 333 (1992).

${ }^{24}$ M. S. Somayazulu, S. M. Sharma, N. Garg, S. L. Chaplot, and S. K. Sikka, J. Phys. C 65, 6345 (1993).

${ }^{25}$ B. Vessal, M. Amini, and H. Akbarzadeh, J. Chem. Phys. 101, 7823 (1994).

${ }^{26}$ W. Jin, R. K. Kalia, P. Vashishta, and J. P. Rino, Phys. Rev. B 50, 118 (1994).

${ }^{27}$ J. Badro, J. L. Barrat, and P. Gillet, Phys. Rev. Lett. 76, 772 (1996).

${ }^{28}$ J. F. Stebbins and P. F. McMillan, Am. Mineral. 74, 965 (1989).

${ }^{29}$ X. Xue, J. F. Stebbins, M. Kanzaki, and R. G. Tronnes, Science 241, 962 (1989).

${ }^{30}$ I. Farnan and J. F. Stebbins, J. Am. Chem. Soc. 112, 32 (1990).

${ }^{31}$ G. H. Wolf, D. J. Durben, and P. F. McMillan, J. Chem. Phys. 93, 2280 (1990).

${ }^{32}$ X. Xue, J. F. Stebbins, M. Kanzaki, P. F. McMillan, and B. Poe, Am. Mineral. 76, 8 (1991).

${ }^{33}$ J. F. Stebbins, Nature (London) 351, 638 (1991).

${ }^{34}$ J. F. Stebbins, I. Farnan, and X. Xue, Chem. Geol. 96, 371 (1992).

${ }^{35}$ P. F. McMillan, B. T. Poe, P. Gillet, and B. Reynard, Geochim. Cosmochim. Acta 58, 3653 (1994).

${ }^{36}$ D. L. Farber and Q. Williams, Am. Mineral. 81, 273 (1996).

${ }^{37}$ R. M. Laine, K. Y. Blohowiak, T. R. Robinson, M. L. Hoppe, P. Nardi, J. Kampf, and J. Uhm, Nature (London) 353, 642 (1991).

${ }^{38}$ B. Herreros, S. W. Carr, and J. Klinowski, Science 263, 1585 (1994)

${ }^{39}$ F. Liebau, Inorg. Chim. Acta 89, 1 (1984).

${ }^{40}$ J. D. Kubicki, Y. Xiao, and A. C. Lasaga, Geochim. Cosmochim. Acta 57, 3847 (1993).
${ }^{41}$ Y. Xiao and A. C. Lasaga, Geochim. Cosmochim. Acta 57, 2283 (1996).

${ }^{42}$ R. E. Cohen, Silica-Physical Behavior, Geochemistry, and Materials Applications, Reviews of Mineralogy (Ref. 1), p. 369.

${ }^{43}$ N. R. Keskar and J. R. Chelikowsky, J. Chem. Phys. 22, 233 (1995).

${ }^{44}$ J. S. Tse, D. D. Klug, and D. C. Allan, Phys. Rev. B 51, 16392 (1995).

${ }^{45}$ K. de Boer, A. P. J. Jansen, and R. A. van Santen, Phys. Rev. B 52, 12579 (1995).

${ }^{46}$ B. van Beest, G. J. Kramer, and R. A. van Santen, Phys. Rev. Lett. 64, 1955 (1990).

${ }^{47}$ H. J. Berendsen, J. P. O. Postma, W. I. van Gunsteren, A. Di Niola, and J. R. Haak, J. Chem. Phys. 81, 3684 (1984).

${ }^{48}$ W. Kohn and L. J. Sham, Phys. Rev. 140, 1133 (1965).

${ }^{49}$ M. P. Teter, M. C. Payne, and D. C. Allan, Phys. Rev. B 40, 12255 (1989).

${ }^{50}$ N. Troullier and J. L. Martins, Phys. Rev. B 43, 1993 (1991).

${ }^{51}$ S. G. Louie, S. Froyen, and M. L. Cohen, Phys. Rev. B 26, 1738 (1982).

${ }^{52}$ H. J. Monkhorst and J. D. Pack, Phys. Rev. B 13, 5188 (1976).

${ }^{53}$ J. S. Tse and D. D. Klug, Phys. Rev. Lett. 67, 3559 (1991).

${ }^{54}$ N. Binggeli and J. R. Chelikowsky, Phys. Rev. Lett. 69, 2220 (1992).

${ }^{55}$ R. T. Downs, K. L. Bartelmehs, G. V. Gibbs, and M. B. Boisen, Am. Mineral. 78, 1104 (1993).

${ }^{56}$ P. E. Werner, L. Eriksson, and J. Westdahl, J. Appl. Crystallogr. 18, 367 (1985).

${ }^{57}$ M. B. Boisen, G. V. Gibbs, and M. S. T. Bukowinski, Phys. Chem. Miner. 21, 269 (1994).

${ }^{58}$ D. Wolff, J. Appl. Crystallogr. 1, 108 (1968).

${ }^{59}$ R. J. Angel, N. L. Ross, F. Seifert, and T. Fliervoet, Nature (London) 384, 441 (1996).

${ }^{60}$ Y. Kudoh and M. Kanzaki, EOS Trans. Am. Geophys. Union 77, F560 (1996).

${ }^{61}$ N. Binggeli, N. Troullier, J. L. Martins, and J. R. Chelikowsky, Phys. Rev. B 44, 4771 (1991).

${ }^{62}$ A. Di Pomoponio and A. Continenza, Phys. Rev. B 50, 5950 (1994).

${ }^{63}$ Y. Xu and W. Y. Ching, Phys. Rev. B 44, 11048 (1991).

${ }^{64}$ J. P. Hansen and I. R. McDonald, Theory of Simple Liquids, 2nd ed. (Cambridge University Press, London, 1989).

${ }^{65}$ G. Dolino and M. Vidale, Silica-Physical Behavior, Geochemistry, and Materials Applications, Reviews of Mineralogy (Ref. 1), p. 403.

${ }^{66}$ F. Birch, J. Geophys. Res. 83, 1257 (1978).

${ }^{67}$ J. D. Jorgensen, J. Appl. Phys. 49, 5473 (1978).

${ }^{68}$ L. Levien and C. T. Prewitt, Am. Mineral. 66, 324 (1981)

${ }^{69}$ R. M. Hazen, L. W. Finger, R. J. Hemley, and H. K. Mao, Solid State Commun. 72, 507 (1989).

${ }^{70}$ J. Glinnemann, H. E. King, H. Schulz, T. Hahn, S. J. La Placa, and F. Dacol, Z. Kristallogr. 198, 177 (1992).

${ }^{71}$ K. J. Kingma, Ph.D. thesis, Johns Hopkins University, 1994.

${ }^{72}$ L.-g. Liu, W. A. Bassett, and T. Takahashi, J. Geophys. Res. 79, 1160 (1974).

${ }^{73}$ N. L. Ross, J. F. Shu, R. M. Hazen, and T. Gasparik, Am. Mineral. 75, 739 (1990).

${ }^{74}$ J. F. Shu, H. K. Mao, and R. J. Hemley (unpublished)

${ }^{75}$ D. M. Teter, G. V. Gibbs, M. B. Boisen, D. C. Allan, and M. P. Teter, Phys. Rev. B 52, 8064 (1995).

${ }^{76}$ J. S. Tse and D. D. Klug, J. Chem. Phys. 95, 9176 (1991). 
${ }^{77}$ N. Binggeli, N. R. Keskar, and J. R. Chelikowsky, Phys. Rev. B 49, 3075 (1994).

${ }^{78}$ G. W. Watson and S. C. Parker, Phys. Rev. B 52, 13306 (1995).

${ }^{79}$ A. G. Christy, Acta Crystallogr. Sect. B 51, 753 (1995).

${ }^{80}$ A. G. Christy and R. J. Angel, Phys. Chem. Miner. 22, 129 (1995).

${ }^{81}$ D. R. Allan, J. S. Loveday, R. J. Nelmes, and P. A. Thomas, J. Phys. Condens. Matter 4, 2747 (1992).
${ }^{82}$ S. Tsuneyuki, Y. Matsui, H. Aoki, and M. Tsukada, Nature (London) 339, 209 (1989).

${ }^{83}$ D. R. Hamann, Phys. Rev. Lett. 76, 660 (1996).

${ }^{84}$ H. K. Mao, J. Shu, Y. Fei, J. Hu, and R. J. Hemley, Phys. Earth Planet. Inter. 96, 135 (1996).

${ }^{85}$ R. J. Hemley, H. K. Mao, G. Shen, J. Badro, P. Gillet, M. Hanfland, and D. Häusermann, Science 276, 1242 (1997). 